\title{
TITLE:
}

\section{Dynamic phase transition in a rotating external field}

\section{$\operatorname{AUTHOR}(S)$ :}

Fujiwara, N; Kobayashi, T; Fujisaka, H

\section{CITATION:}

Fujiwara, N ...[et al]. Dynamic phase transition in a rotating external field. Physical Review E 2007, 75(2): 026202.

ISSUE DATE:

2007-02

URL:

http://hdl.handle.net/2433/50557

RIGHT:

Copyright 2007 American Physical Society 
PHYSICAL REVIEW E 75, 026202 (2007)

\title{
Dynamic phase transition in a rotating external field
}

\author{
Naoya Fujiwara, ${ }^{*}$ Takeo Kobayashi, ${ }^{\dagger}$ and Hirokazu Fujisaka ${ }^{\ddagger}$ \\ Department of Applied Analysis and Complex Dynamical Systems, Graduate School of Informatics, Kyoto University, \\ Kyoto 606-8501, Japan \\ (Received 18 September 2006; published 2 February 2007)
}

\begin{abstract}
Dynamic phase transition in the Ginzburg-Landau model of the anisotropic $X Y$ spin system in a rotating external field is studied. We observe several types of oscillations, limit cycles, quasiperiodic oscillations and chaotic motions. It is found that limit cycle oscillations can have the periodicity of multiple times of the period of the applied field and that the system shows two kinds of scenarios leading to the onset of quasiperiodic oscillations, i.e., the saddle-node and Hopf bifurcations. Furthermore, this paper reports the findings of chaotic behaviors in the context of dynamic phase transition and that there exist two types of chaos with and without a certain kind of symmetry.
\end{abstract}

DOI: 10.1103/PhysRevE.75.026202

PACS number(s): 05.45.-a, 64.60.-i, 82.40.Bj

\section{INTRODUCTION}

Dynamic phase transition (DPT) is an example of the nonequilibrium phase transition in the presence of the temporally oscillating external field $H(t)$ which has usually the symmetry $H(t)=-H(t+T / 2), T$ being its period. Temporal oscillation of the response $M(t)$ can be classified into two types according to its symmetry. One is the symmetryrestoring oscillation (SRO) which holds the symmetry $M(t)=-M(t+T / 2)$, and the other the symmetry-breaking oscillation (SBO) without this symmetry. The transition between SRO and SBO states under the change of the frequency or the amplitude of the external field is called DPT. In a magnetic system, $H(t)$ and $M(t)$ correspond to the magnetic field and the magnetization, respectively. After its discovery [1], DPT has been extensively studied in the frameworks of the mean field theory [1-7], Monte Carlo simulation [3,8-13], and laboratory experiments [14,15]. Related works in the ferromagnetic systems are reviewed in Refs. [16,17]. Recently, DPTs have been investigated in various physical systems such as in the $\mathrm{CO}$ oxidation process on a catalytic surface by changing the partial pressure of $\mathrm{CO}$ [18] and in two-dimensional fully frustrated Josephsonjunction arrays [19]. Associated with DPT, a method to stabilize the unstable SRO in SBO regime has been proposed [20].

The deterministic nature of the temporally oscillating force plays a crucial role for DPT. This implies that the deterministic models capture the characteristics of DPT. Fujisaka, Tutu, and Rikvold studied the DPT in the Ising model below the critical temperature $T_{c}$ using the timedependent Ginzburg-Landau (TDGL) equation in a periodic external force

$$
\begin{gathered}
\dot{S}(\boldsymbol{r}, t)=\Delta T S-S^{3}+D \nabla^{2} S+h \cos (\Omega t), \\
\left(\Delta T \equiv T_{c}-T_{\text {temp }}>0\right)
\end{gathered}
$$

from the viewpoint of the deterministic mean field theory.

\footnotetext{
*Electronic address: naoya@acs.i.kyoto-u.ac.jp

†Electronic address: takeo@acs.i.kyoto-u.ac.jp

‡Electronic address: fujisaka@i.kyoto-u.ac.jp
}

Here, $T_{\text {temp }}$ is the system temperature and $S(\boldsymbol{r}, t)$ is identical to the local magnetization at location $\boldsymbol{r}$ and time $t$ [4]. They found that DPT is equivalent to the bifurcation observed as either $h$ or $\Omega$ is changed. They have shown that DPT belongs to the universality class of the equilibrium phase transition in the two-dimensional Ising model. This fact has been confirmed with the Monte Carlo studies of two-dimensional Ising model in an oscillating magnetic field [9-11,16].

Equation (1) can be extended to the anisotropic $X Y$ model

$$
\dot{\psi}(\boldsymbol{r}, t)=\Delta T \cdot \psi-|\psi|^{2} \psi+\gamma \psi^{*}+\nabla^{2} \psi+H(t),
$$

where the complex variable $\psi(\boldsymbol{r}, t)=X(\boldsymbol{r}, t)+i Y(\boldsymbol{r}, t)$ is the order parameter whose real and imaginary parts denote, respectively, the $X$ and $Y$ components, and $H(t)$ denotes the periodic external field which holds the symmetry $H(t)=-H(t$ $+T / 2$ ). The real coefficient $\gamma$ represents the magnitude of the anisotropy. Yasui et al. studied DPTs of the uniform solution in a linearly polarized external field $H(t)=h \cos (\Omega t)$ [5]. They found a new DPT which causes the emergence of a component perpendicular to the external field. This type of DPT has been recently reported with Monte Carlo study in two-dimensional anisotropic Heisenberg spin system in a linearly polarized magnetic field [21]. Furthermore, Fujiwara et al. studied the domain wall dynamics in Eq. (2) for $H(t)$ $=h \cos (\Omega t)[22]$.

Studies of DPT in the multicomponent systems suggest that there exist new types of DPTs if we change type of the external field. Acharyya reported DPTs between three different phases in a uniaxially anisotropic Heisenberg model in an elliptically polarized external field using the Monte Carlo method [23]. In the present paper, we consider the mean-field equation with a rotating external force $H(t)=h e^{i \Omega t}$ and show that various DPTs occur. After a suitable rescaling of variables, Eq. (2) is rewritten as

$$
\dot{\psi}(\boldsymbol{r}, t)=\psi-|\psi|^{2} \psi+\gamma \psi^{*}+\nabla^{2} \psi+h e^{i \Omega t} .
$$

We consider the uniform solutions of this equation throughout this paper.

The paper is organized as follows. In Sec. II, we discuss the symmetry of Eq. (3) which plays an important role for DPTs. In Sec. III, we show numerical results for DPTs of the 
uniform solution. Periodic oscillations are classified by their periods and the symmetry. Furthermore, it will be shown that quasiperiodic oscillation and chaotic oscillation are also observed. This paper is the first report of the observation of quasiperiodic oscillation and chaos in the context of DPT. There exist two types of chaotic attractors. One has a certain symmetry which is associated with that of the external field, and the other does not have this symmetry. We will observe the symmetry breaking of the chaotic attractor with the change of either the frequency or the amplitude of the external field. We will show five fundamental phase diagrams in the $\Omega-h$ plane for different values of the anisotropy parameter $\gamma$. The results are summarized in Sec. IV.

\section{CHARACTERISTICS OF THE MODEL}

In this section, paying attention to the spatially uniform oscillation of Eq. (3), we will study the following equation of motion:

$$
\dot{\psi}(t)=\psi-|\psi|^{2} \psi+\gamma \psi^{*}+h e^{i \Omega t} .
$$

The real and imaginary parts of $\psi(t)=X(t)+i Y(t)$ obey the equations of motion,

$$
\begin{aligned}
& \dot{X}(t)=(1+\gamma) X-\left(X^{2}+Y^{2}\right) X+h \cos (\Omega t), \\
& \dot{Y}(t)=(1-\gamma) Y-\left(X^{2}+Y^{2}\right) Y+h \sin (\Omega t) .
\end{aligned}
$$

Since Eqs. (5) and (6) are invariant under the transformation $\gamma \rightarrow-\gamma, X \rightarrow-Y, Y \rightarrow X, t \rightarrow t+T / 4$, we choose as $\gamma>0$ in the present paper. The period of the external force is given by $T=2 \pi / \Omega$. The insertion of $\psi=\rho(t) e^{i \Omega t+i \phi(t)}$ with the amplitude $\rho$ and the phase $\phi$ into Eq. (4), we obtain the evolution equations

$$
\begin{gathered}
\dot{\rho}=\rho-\rho^{3}+\gamma \rho \cos [2(\Omega t+\phi)]+h \cos \phi, \\
\Omega+\dot{\phi}=-\gamma \sin [2(\Omega t+\phi)]-\frac{h \sin \phi}{\rho} .
\end{gathered}
$$

One easily finds that Eq. (4) is invariant under the transformation

$$
t \rightarrow t+n T / 2, \quad \psi \rightarrow-\psi,
$$

where $n$ is an odd number. Because of this symmetry of the fundamental equations of motion, we expect that there exist trajectories satisfying the symmetry

$$
\psi(t)=-\psi(t+n T / 2) \quad(n \text { :odd }) .
$$

In the remainder of the paper, the motion with the symmetry (10) is called the symmetry-restoring oscillation and the motion without this symmetry the symmetry-breaking oscillation. From the definition of SRO, if it exists, we should have the period with an odd number multiple of the period of the applied field. On the other hand, the period of the SBO limit cycle can be an either even or odd number multiple of the period of the applied field. For the limit cycle with the period $T_{p}, \psi(t)$ satisfying the symmetry (10), the time average

$$
\bar{\psi} \equiv \frac{1}{T_{p}} \int_{0}^{T_{p}} \psi(s) d s
$$

vanishes for SRO and is finite for SBO. The limit cycle satisfying

$$
\begin{gathered}
\psi(t) \neq \psi(t+k T) \quad(k=1,2, \ldots, n-1), \\
\psi(t)=\psi(t+n T),
\end{gathered}
$$

is called a period- $n$ limit cycle. A period- $n$ limit cycle with (without) the symmetry (10) is called the period- $n$ SRO (SBO) and is denoted as SRO- $n$ (SBO- $n$ ).

The phase $\theta(t)$ of the external field at time $t$, defined as $0 \leq \theta(t)<2 \pi$, is given by

$$
\theta(t)=[\theta(0)+\Omega t] \bmod 2 \pi,
$$

and satisfies the relation

$$
\theta(t+k T)=\theta(t) \quad(k=0,1,2, \ldots, n-1) .
$$

Therefore, for a same phase of the external field, there can exist $n$ different state points on the trajectory of a period- $n$ limit cycle. By noting that these states depend on the phase $\theta$ of the external field, they are written as $\psi_{k}\{\theta\}(k$ $=1,2, \ldots, n)$, which satisfy

$$
\begin{aligned}
\psi_{n}\{\theta(t)\} & =\psi_{n-1}\{\theta(t+T)\} \\
& =\cdots=\psi_{1}\{\theta(t+(n-1) T)\}=\psi_{n}\{\theta(t+n T)\} .
\end{aligned}
$$

Because of the symmetry under the transformation (9), if the SBO $\psi^{+}(t)$ exists, then the limit cycle $\psi^{-}(t)$ with the relation $\psi^{-}(t)=-\psi^{+}(t+T / 2)$ also exists. The limit cycle attractor whose time average of the $X$ component is positive is denoted by $\psi^{+}$and that with the negative time average by $\psi^{-}$. We thus find that, there exist two limit cycle attractors $\psi^{ \pm}$in SBO- $n$, on which there exist $2 n$ state points $\psi \psi_{k}^{ \pm}\{\theta\}(k$ $=1,2, \ldots, n)$ for the phase $\theta$ of the external field.

\section{DYNAMIC PHASE TRANSITIONS AMONG VARIOUS DYNAMICAL PHASES}

In this section we show the characteristics of the temporal oscillation of the dynamical phases and DPTs between them. Equation (4) was numerically integrated using the fourthorder Runge-Kutta method with the time step $\Delta t=T / 2000$ for $\Omega \leq 0.5$ and $\Delta t=T / 1000$ for $\Omega>0.5$. Depending on the anisotropy parameter $\gamma$, the system (4) exhibits several types of oscillations, period- 1 and period- $n$ limit cycles ( $n$ $=2,3, \ldots)$, quasiperiodic oscillation and chaos, with different symmetry of attractors. One observes that there exist five fundamental characteristic phase diagrams depending on the value of the anisotropy parameter $\gamma$.

For any parameter values of $\gamma$ (except $\gamma=0)$ and $\Omega$, we observe SBO-1 for sufficiently small value of $h$ and SRO-1 for sufficiently large value of $h$. These facts can be explained as follows. First consider the case $h=0$, for which the system has two fixed point attractors since the system under consid- 
eration is below the critical temperature and the symmetry of the states is broken. Depending on the initial condition, one of these fixed points is chosen. If a weak field is applied to the broken-symmetry state, the system shows a weak oscillation whose amplitude is proportional to $h$ and its frequency is equal to $\Omega$. This oscillation is identical to SBO-1. As the amplitude $h$ is increased, the amplitude of oscillation increases, showing temporally broken oscillations. From the symmetry (9), there exist two limit cycle oscillations except $\gamma=0$. As $h$ is further increased, these two attractors come to merge, which leads to a symmetric oscillation. This is identical to SRO. Since the oscillation is synchronized to the applied field, the system shows SRO-1 for large $h$.

The present system with Eqs. (7) and (8) is regarded as the self-oscillatory system superimposed by the external force with the period $T / 2$. Therefore, SRO and SBO trajectories are regarded as the synchronized motions and the quasiperiodic oscillation as the nonsynchronized oscillation to the external force. Let $\Omega^{\prime}$ be the frequency of $\rho(t)$ and $\phi(t)$. Since $\rho(t)=\rho(t+T / 2), \phi(t)=\phi(t+T / 2)$ for $\Omega^{\prime}=2 \Omega$, we obtain

$$
\psi(t)=-\psi(t+T / 2) .
$$

This relation implies that the trajectory under consideration turns out to be an SRO-1 trajectory. On the other hand, since $\psi(t) \neq-\psi(t+T / 2)$ for $\Omega^{\prime}=\Omega$, the oscillation corresponds to the SBO trajectory. Generally, if $\Omega$ is a half-odd number multiple of $\Omega^{\prime}$, the limit cycle oscillation is identical to SRO and otherwise it is SBO. In the following, we will give five typical phase diagrams for different values of the anisotropy parameter $\gamma$.

\section{A. Phase diagram 1: Period-1 limit cycles}

Numerical integration was carried out for a strong anisotropy, i.e., for large values of $\gamma$ with $\gamma=0.6$. In this case, we observe two types of limit cycle oscillations, SRO-1 and SBO-1. Figures 1(a) and 1(b) are, respectively, the projections of trajectories of SRO-1 and SBO-1 onto the $X Y$ plane. In each figure, the state points move in the anticlockwise way. The SRO trajectory satisfies the relation (10) and has a rotational symmetry around the origin $X=Y=0$. In the SBO phase, there exist two limit cycles $\psi^{+}$and $\psi^{-}$, one of which is observed according to the initial condition. For a strong anisotropy, only DPT between SRO-1 and SBO-1 is observed. Since the system under consideration is generated by the periodic external field, there exists no zero-value Floquet exponent except the quasi-periodic case. The Floquet exponent vanishes at the DPT points. The phase diagram drawn in Fig. 2 was determined by observing vanishing points of the Floquet exponent as the parameter, either $h$ or $\Omega$, is changed.

\section{B. Phase diagram 2: Period-2 limit cycles}

For small values of the anisotropy parameter $\gamma$, we observe limit cycle oscillations with period longer than the period of the applied external field. For anisotropy parameter value, e.g., $\gamma=0.55$, we fundamentally observe SRO- 1 similar to those shown in Fig. 1(a) and SBO-1 in Fig. 1(b). In
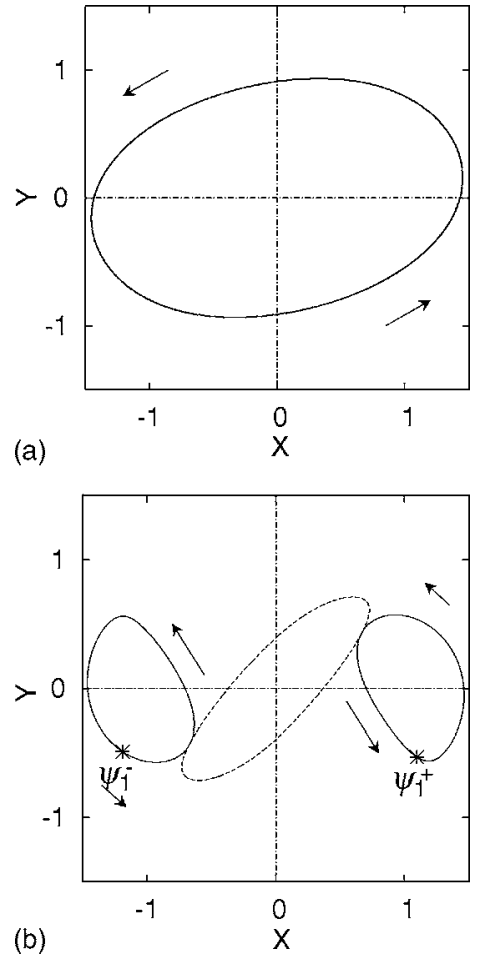

FIG. 1. (a) The SRO-1 trajectory for $\gamma=0.6, \Omega=1.0$, and $h$ $=1.5$. (b) The SBO- 1 trajectory for $\gamma=0.6, \Omega=1.0$, and $h=0.86$. There exist two trajectories $\psi^{+}$and $\psi^{-}$. State points $\psi_{1}^{+}\{0\}$ and $\psi_{1}^{-}\{0\}$ denoted by the symbol $*$ are equal to the values of $\psi(t)$ for the phase $\theta(t)=0$ of the external force. The dotted curve in (b) represents the unstable SRO-1.

addition, there exists SBO-2 shown in Fig. 3 in a certain region of $h$ and $\Omega$. For a given phase $\theta$ of the external force, there exist four state points, i.e., two points $\psi_{1,2}^{+}$on the trajectory $\psi^{+}$and $\psi_{1,2}^{-}$on the trajectory $\psi^{-}$. Due to Eq. (15), they are related to each other via

$$
\psi_{2}^{ \pm}\{\theta(t)\}=\psi_{1}^{ \pm}\{\theta(t+T)\}=\psi_{2}^{ \pm}\{\theta(t+2 T)\} .
$$

For $\gamma=0.55$, there exists DPT between SBO- 1 and SBO-2 which associates with the change of the period of limit

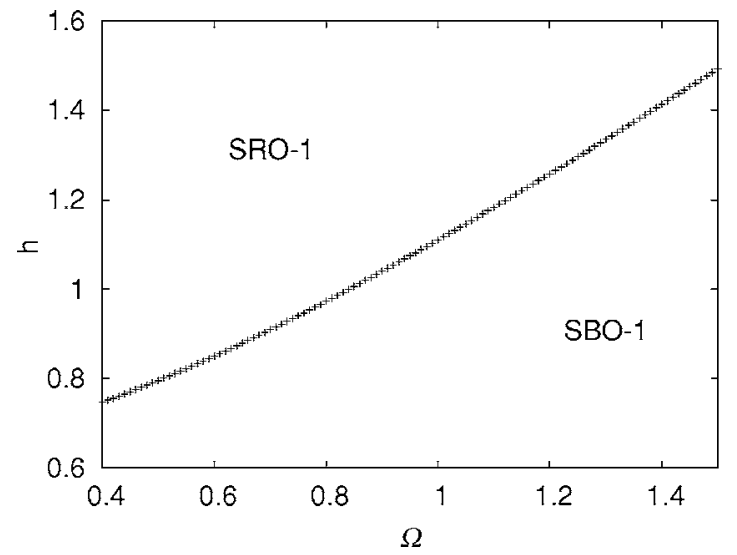

FIG. 2. Phase diagram for $\gamma=0.6$. DPT between SRO-1 and SBO-1 is observed. 


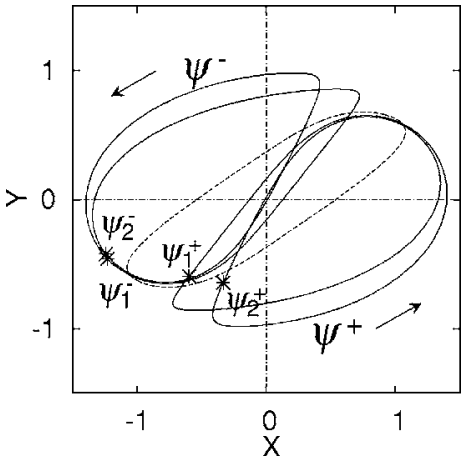

FIG. 3. SBO-2 trajectories for $\gamma=0.55, \Omega=0.65$, and $h=0.81$. There exist two attractors corresponding to trajectories $\psi^{+}$and $\psi^{-}$. The state points $\psi_{1}^{+}, \psi_{2}^{+}, \psi_{1}^{-}$, and $\psi_{2}^{-}$represent all the values of $\psi\{\theta=0\}$ on the attractor, corresponding to the phase $\theta=0$. The dotted curve stands for the unstable SRO-1 trajectory.

cycles in addition to DPT between SRO- 1 and SBO- 1 which brings the change of the symmetry of oscillation as for $\gamma$ $=0.6$. Since the largest Floquet exponent vanishes at the bifurcation point between SBO-1 and SBO-2, we can determine their transition point. Figure 4 shows the bifurcation diagram and the largest Floquet exponent drawn as a function of the strength $h$ of the applied field for $\gamma=0.55$ and $\Omega=0.5$. In Fig. 4(a), phase points in $\psi^{+}$are drawn. In addition to them, there exist phase points belonging to $\psi^{-}$. However, they are not shown in the figure. Here the quantity $X_{m}$ in Fig. 4(a) is defined as
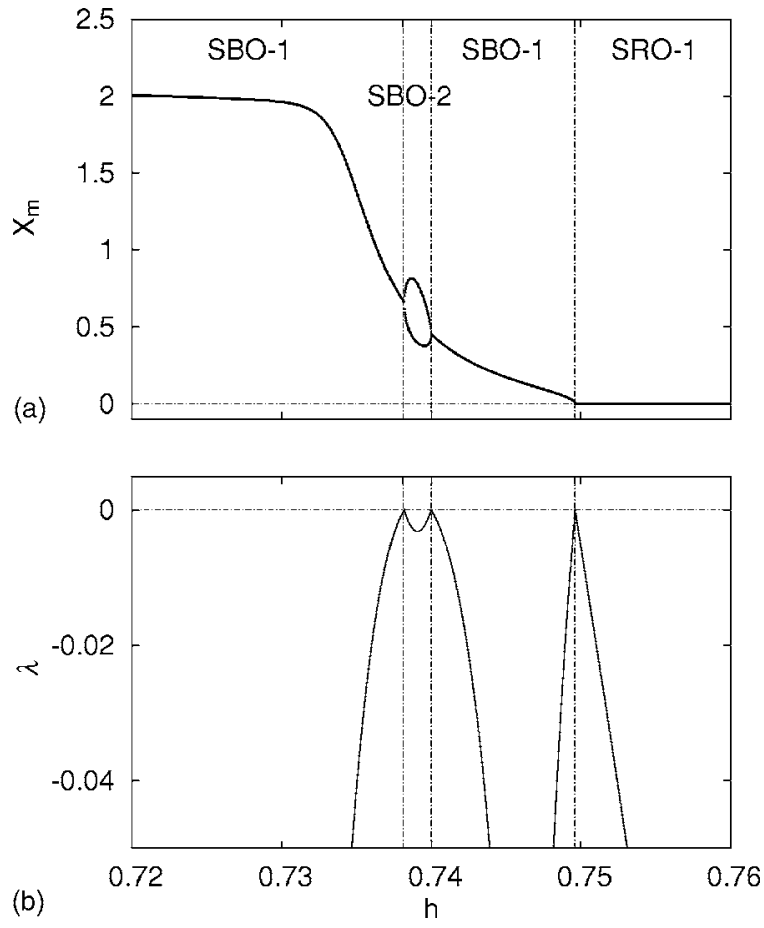

FIG. 4. (a) Bifurcation diagram and (b) the largest Floquet exponent $\lambda$ for $\gamma=0.55$ and $\Omega=0.5$. The bifurcation point is determined by that where the largest Floquet exponent vanishes. For the definition of $X_{m}$, see the text.

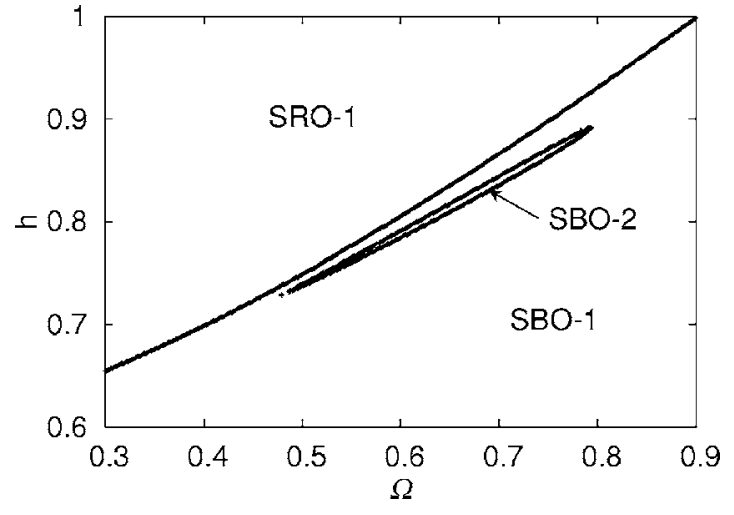

FIG. 5. Phase diagram for $\gamma=0.55$. There exist two dynamical phases SBO-1 and SBO-2 in the SBO region.

$$
X_{m} \equiv X\left(t_{0}\right)+X\left(t_{0}+\frac{(2 m+1) T}{2}\right) \quad(m=0,1,2, \ldots)
$$

the time origin $t_{0}$ being chosen as the time when $X$ has the maximum value $X\{\theta=0\}$. The $X_{m}$ 's in Figs. 7, 8, and 14 are defined in the same way as the above. For this selection of $t_{0}$, we find $X_{m} \geq 0$ for SRO. If the oscillation is a period- $n$ trajectory, i.e., $X_{n}=X_{0}$, then we have $n$ phase points, $\left\{X_{0}, X_{1}, \ldots, X_{n-1}\right\}$. For SRO- $n$, because of the relation (10), we find $X_{(n-1) / 2}=0$. On the other hand, the relation (10) does not hold for SBO- $n$. Thus, one can distinguish SRO and SBO by observing whether a state point in the bifurcation diagram satisfy $X_{m}=0$ for $m=(n-1) / 2$. The periodicity of the oscillation is found by observing the number of the points. Since commonly used period-averaged order parameter does not give information on the period of a limit cycle, the bifurcation diagrams in this paper are plotted as described above.

The largest Floquet exponent $\lambda$ is drawn in Fig. 4(b). The bifurcation point is determined by that where $\lambda$ vanishes. The phase diagram obtained from the Floquet analysis is shown in Fig. 5. As shown above, for $\gamma=0.55$, there exists the SBO-2 phase for the frequency region $0.49 \leq \Omega \leq 0.79$ in the SBO phase. The bifurcation diagram Fig. 4(a) for $\gamma$ $=0.55$ and $\Omega=0.5$ shows that there exist SRO- 1 for $h$ $\geq 0.7496$, SBO- 1 for $0.74 \leqq h \leqq 0.7496$ and $h \leqq 0.7381$, and SBO-2 for $0.7381 \leqq h \leqq 0.74$.

Our basic time evolution equation (4) has three variables, $X, Y$, and $t$. For $\gamma$ is sufficiently large, the effective degree of freedom is reduced to two because the $Y$ component is so small that it does not influence on the dynamics of $X$. The time evolution for $\gamma=0$ can be described by two variable equations (19) and (20), which will be discussed in Sec. III D. Since the number of effective degrees of freedom reduces in the limits of both small and large $\gamma$, chaotic oscillation is observed only in the intermediate range of $\gamma$ value. As $\gamma$ is further decreased below 0.55 , the system shows a period doubling bifurcation and SBOs with long periodicity are observed. For $\gamma \gtrsim 0.4$, only the limit cycle oscillations exist.

\section{Phase diagram 3: Chaos}

We numerically integrated the system for an intermediate value of the anisotropy parameter, $\gamma=0.3$, for which there 
exist several types of complex motions. Particularly, we observed chaotic oscillation for $\Omega=0.5$ [Figs. 6(a) and 6(b)]. This is the first observation of chaotic phase in the context of the dynamic phase transition. The Lyapunov exponents for the trajectories in Figs. 6(a) and 6(b) are found to be positive. Since the chaotic attractor in Fig. 6(a) is symmetric around the origin, the long time average of $\psi(t)$ vanishes. The symmetry of the governing equation (4) implies that if the state point $\psi\{\theta\}$ for the phase $\theta$ is on the attractor, then $-\psi\{\theta$ $+\pi\}$ is also on the same attractor. This type of chaotic trajectory will be called the symmetry-restoring chaos (SRC). On the contrary, the chaotic motion shown in Fig. 6(b) is not symmetric in the sense that the long time average of $\psi$ does not vanish. This type of chaos will be called the symmetrybreaking chaos (SBC). From the symmetry, there exist two SBC attractors. One of them $\left(\psi^{+}\right)$is drawn in Fig. 6(c). Figure 6(d) represents the SRO-3 trajectory.

The bifurcation diagram and the largest Lyapunov exponent for $\gamma=0.3$ and $\Omega=0.5$ are shown in Fig. 7. The largest Lyapunov exponent takes a positive value in the chaotic phase. One observes SRO- 1 for $h \geq 0.5553$, chaotic motion for $0.5406 \leqq h \leqq 0.5517$ and in a small region near $h$ $=0.5025$, SRO- 3 for $0.5025 \leqq h \leqq 0.5387$, SBO-3 for $0.5387 \leqq h \leqq 0.5406$ and SBO- 1 for $h \leqq 0.499$.

Figure 8(a) shows the enlargement of Fig. 7(a). In this parameter region, chaotic motion sets in through the perioddoubling bifurcation. It should be noted that the long time average of $X(t)$ vanishes for SRC. Therefore, by observing the distribution of $X_{m}$ for large $m$, one can determine whether chaos in Figs. 7 and 8 belong to either SRC or SBC. When two SBC attractors merge, the symmetry of the dynamics comes to recover and SRC is observed. This phenomenon is an example of the attractor merging crisis [24]. The long time average of $X(t)$ is calculated and the result is shown in Fig. 8(b).

The phase diagram is shown in Fig. 9. Transition points are again determined as the vanishing points of Floquet exponents of limit cycles. For $\gamma=0.3$, there exists SBO-2 for a certain range of $\Omega$. This situation is similar to that for $\gamma$ $=0.55$. Furthermore, in contrast to that for $\gamma=0.55$, there exist chaos and SRO-3 in the inner side of the SBO-2 region. The phase diagram is more complex than for $\gamma=0.55$.

\section{Phase diagram 4: Quasiperiodic motion for $\gamma=0$ (the isotropic $X Y$ model)}

For the isotropic case, i.e., for $\gamma=0$, it is numerically found that there exist DPT between SRO-1 and quasiperiodic motion (QP). In this case, we can analytically obtain the SRO solution and determine the transition line, examining the linear stability of the SRO solution. This is done as follows. The insertion of $\psi(t)=\rho(t) e^{i \Omega t+i \phi(t)}$ into Eq. (4) leads to the equations of motion for $\rho(t)$ and $\phi(t)$ as

$$
\begin{gathered}
\dot{\rho}=\rho-\rho^{3}+h \cos \phi, \\
\dot{\phi}=-\Omega-\frac{h \sin \phi}{\rho} .
\end{gathered}
$$

Since these equations are autonomous, possible attractor is a fixed point or a limit cycle. From these equations, we obtain
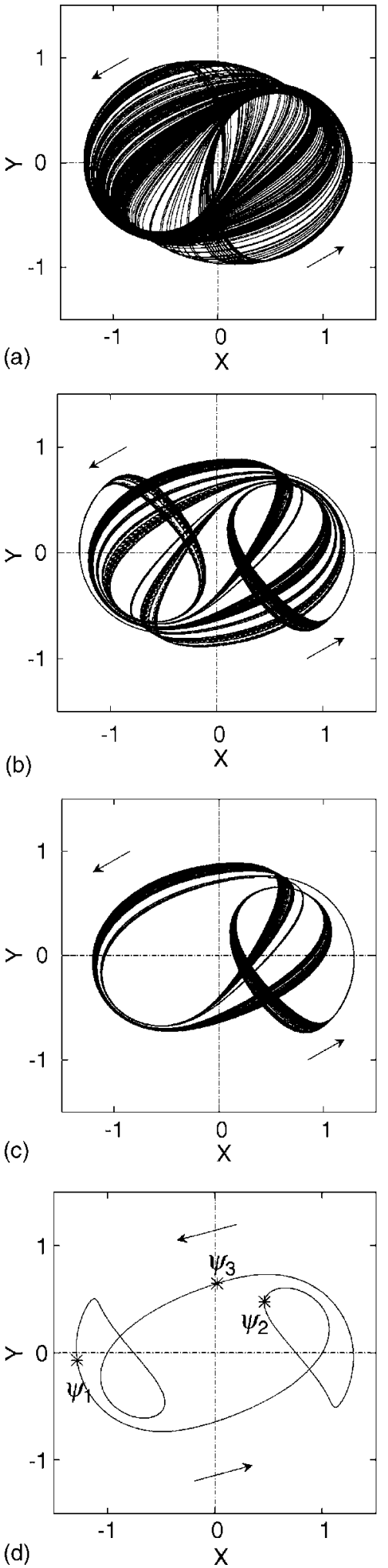

FIG. 6. Chaos and SRO-3 trajectories observed for $\gamma=0.3$. (a) The SRC trajectory for $\Omega=0.5$ and $h=0.545$ over $400 T$. The attractor is statistically symmetric around the origin. (b) The SBC trajectory for $\Omega=0.5$ and $h=0.5415$. The figure shows the coexistence of two chaotic attractors corresponding to $\psi^{+}$(solid curve) and $\psi^{-}$ (dotted curve). The trajectory $\psi^{+}$is shown in (c). (d) The SRO-3 trajectory for $\Omega=0.5$ and $h=0.52$. There exist three state points $\psi_{1}$, $\psi_{2}$, and $\psi_{3}$ for the same phase $\theta(t)$ of the external force. 

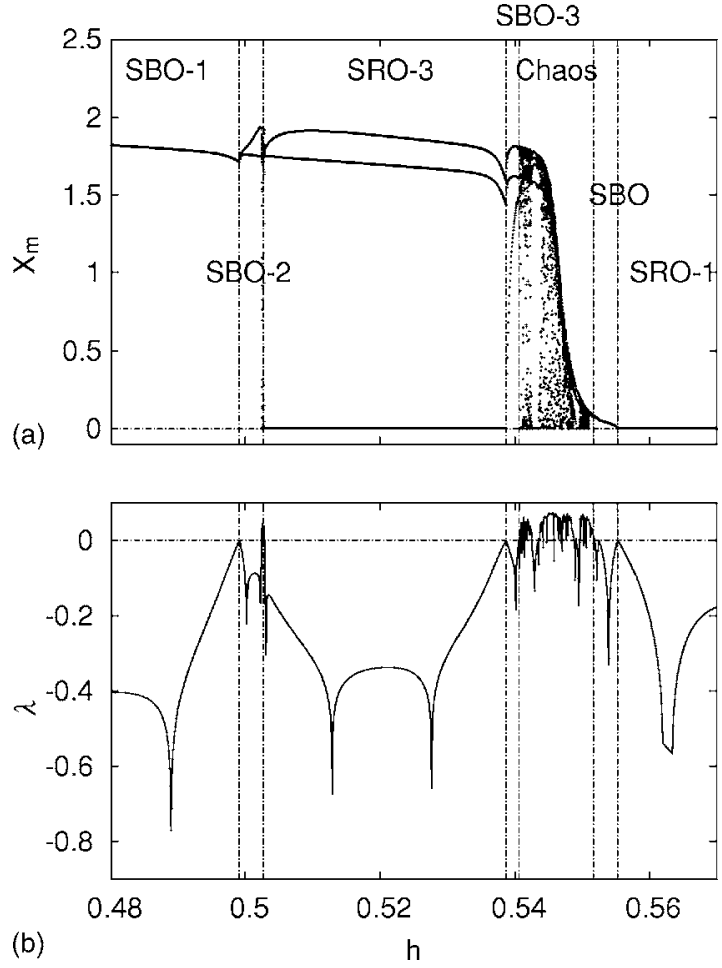

FIG. 7. (a) Bifurcation diagram and (b) the largest Lyapunov exponent $\lambda$ vs the strength of the external field $h$ for $\gamma=0.3$ and $\Omega=0.5$. The definition of $X_{m}$ is the same as in Fig. 4(a).

$$
\left[\dot{\rho}-\left(\rho-\rho^{3}\right)\right]^{2}+\rho^{2}(\dot{\phi}+\Omega)^{2}=h^{2} .
$$

The fixed point solution $\rho_{0}(h, \Omega)$ and $\phi_{0}(h, \Omega)$ of Eqs. (19) and (20) is determined by

$$
\begin{gathered}
h^{2}=f\left(\rho_{0}^{2}\right), \quad f(\xi) \equiv \xi\left[\Omega^{2}+(1-\xi)^{2}\right], \\
h \sin \phi_{0}=-\rho_{0} \Omega .
\end{gathered}
$$

This solution represents the limit cycle of $\psi(t)$ with the period $T$ same as the external force, having the phase lag $\left(-\phi_{0}\right)$ in comparison with the phase of the external force. Since this motion has the symmetry $\psi(t)=-\psi(t+T / 2)$, it belongs to SRO-1. The $f\left(\rho_{0}^{2}\right)$ is a cubic function of $\rho_{0}^{2}$ and provides either one or three fixed point solutions for $\Omega$ $<1 / \sqrt{3}=0.57735 \ldots$ and one fixed point for $\Omega>1 / \sqrt{3}$.

When the above fixed point in a rotating coordinate system is unstable, a limit cycle oscillation comes into existence. Its frequency $\Omega^{\prime}$ and the external frequency $\Omega$ are generally incommensurate. This means that the limit cycle solution in the rotating system corresponds to a quasiperiodic oscillation of $\psi(t)$. In this way, for $\gamma=0$ possible oscillations are SRO-1 and QP. The transition point between SRO-1 and QP can be determined by the linear stability analysis of SRO-1 as shown in Appendix A. The resulting phase diagram is shown in Fig. 10. There are two types of instability of SRO-1 leading to the onset of QP of $\psi(t)$, i.e., the Hopf bifurcation and the saddle-node bifurcation. As $h$ is decreased from sufficiently large $h$, for
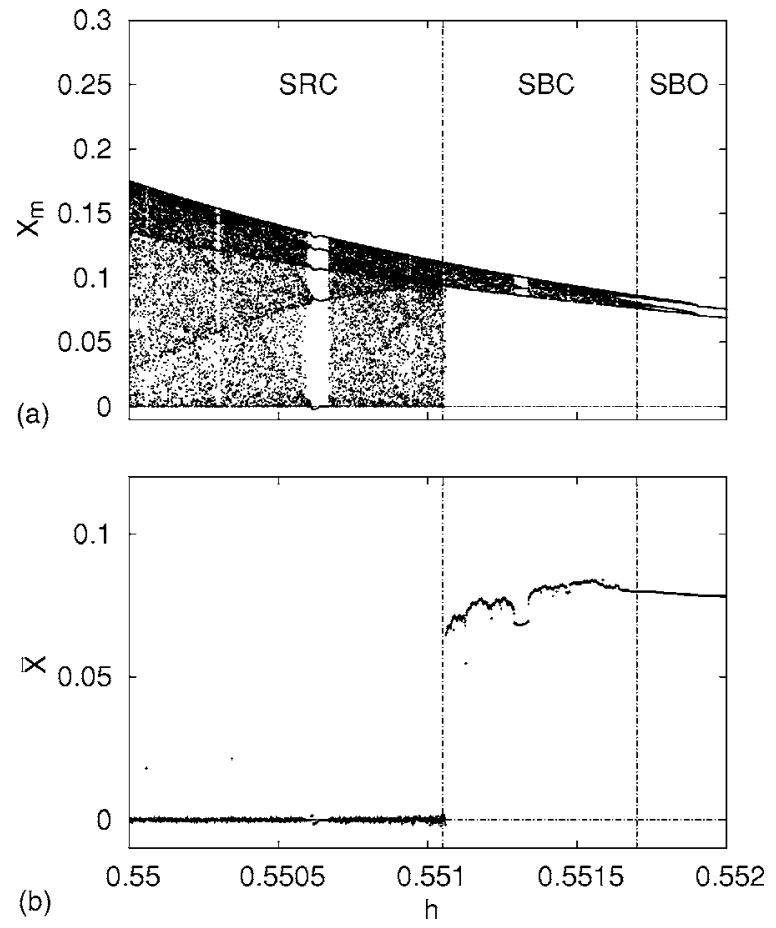

FIG. 8. (a) Enlargement of the bifurcation diagram in Fig. 7. In the SBC and SBO regions, only the phase points belonging to $\psi^{+}$ are drawn. (b) Time average $\bar{X}$ of $X(t)$ is obtained by $\bar{X}$ $=(N T)^{-1} \int_{0}^{N T} X(t) d t,\left(N=10^{4}\right)$, where $X(t)$ is the real part of $\psi(t)$ belonging to $\psi^{+}$. One clearly observes the transition between SRC and SBC.

$$
\Omega<\Omega_{c} \equiv \frac{\sqrt{5}}{4}=0.55901 \ldots
$$

the stable and unstable SRO-1 fixed points collide and disappear at $h=h_{\mathrm{SN}}^{(1)}$ (saddle-node bifurcation). Here, the bifurcation point is given by

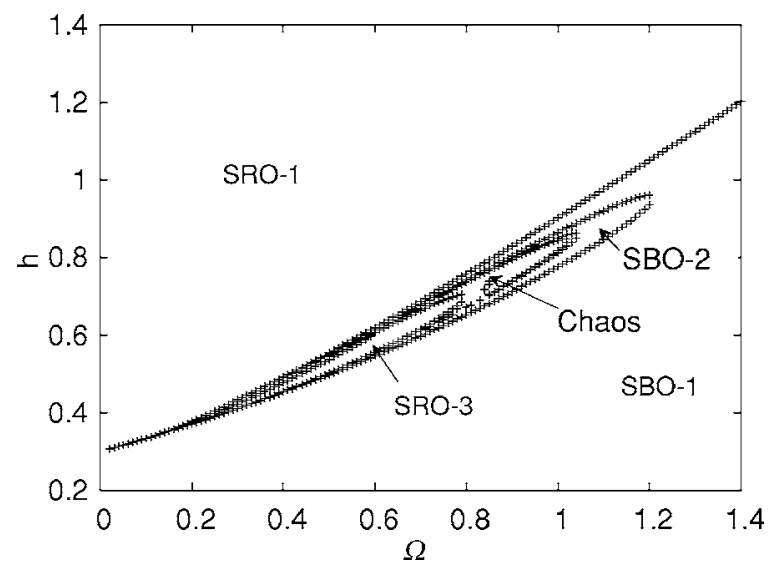

FIG. 9. Phase diagram for $\gamma=0.3$. One finds various dynamical phases including chaos and SRO-3. 

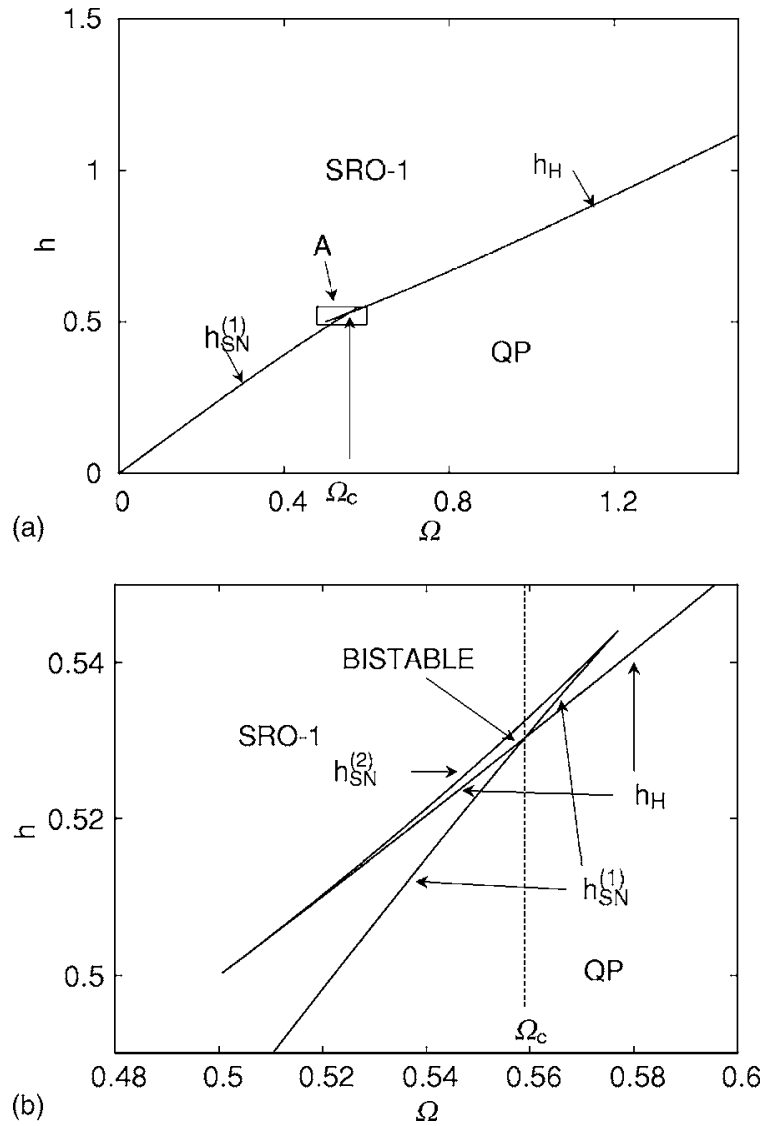

FIG. 10. (a) Phase diagram for the isotropic case $(\gamma=0)$. Transition occurs at the boundary between SRO-1 and QP. Enlargement of the region $A$ is shown in (b). In the region denoted by "BISTABLE" for $1 / 2<\Omega<1 / \sqrt{3}$, there exist two stable SRO-1 solutions.

$$
h_{\mathrm{SN}}^{(1)}(\Omega)=\sqrt{\frac{2+\sqrt{1-3 \Omega^{2}}}{3}\left[\Omega^{2}+\left(\frac{1-\sqrt{1-3 \Omega^{2}}}{3}\right)^{2}\right]} .
$$

Particularly, we obtain $h_{\mathrm{SN}}^{(1)}=\Omega$ for $\Omega \ll 1 / \sqrt{3}$. On the other hand, for $\Omega>\Omega_{c}$, as $h$ is decreased, the SRO-1 phase loses its stability. Examining the linear stability of the fixed point, one finds that this instability is the Hopf bifurcation at the bifurcation point

$$
h_{H}(\Omega)=\sqrt{\frac{4 \Omega^{2}+1}{8}} .
$$

For $h<h_{H}(\Omega)$ a quasiperiodic motion of $\psi(t)$ is observed.

The global phase diagram is shown in Fig. 10(a). Furthermore, a detailed analysis given in Appendix A shows that there exist regions where the bistability of two SRO-1 solutions is observed in the regions $h_{H}<h<h_{\mathrm{SN}}^{(2)}$ for $1 / 2<\Omega$ $<\Omega_{c}$ and $h_{\mathrm{SN}}^{(1)}<h<h_{\mathrm{SN}}^{(2)}$ for $\Omega_{c}<\Omega<1 / \sqrt{3}$ [Fig. 10(b)], where

$$
h_{\mathrm{SN}}^{(2)}=\sqrt{\frac{2-\sqrt{1-3 \Omega^{2}}}{3}\left[\Omega^{2}+\left(\frac{1+\sqrt{1-3 \Omega^{2}}}{3}\right)^{2}\right]} .
$$

Figures 11(a) and 11(c) display the trajectories in the $X Y$ plane of quasiperiodic motion, respectively, below the saddle-node and Hopf bifurcation transition points. It is found that slightly below the saddle-node bifurcation point, one observes an intermittent evolution of amplitude [Fig. 11(b)]. The period $\tau$ of $\rho$ and $\phi$ tends to diverge as $h$ is approached to $h_{\mathrm{SN}}^{(1)}$, and is evaluated as follows. As shown in Appendix B, the slow evolution of $\phi$ near $\phi=\phi_{\mathrm{SN}}$, i.e., the fixed point phase at $h=h_{\mathrm{SN}}^{(1)}$, for $h<h_{\mathrm{SN}}^{(1)}$ is evaluated as

$$
\dot{\phi}(t)=-a\left(h_{\mathrm{SN}}^{(1)}-h\right)-b\left(\phi-\phi_{\mathrm{SN}}\right)^{2},
$$

where $a$ and $b$ are positive constants. The characteristic time to escape from the region $\left|\phi-\phi_{\mathrm{SN}}\right| \sim \sqrt{h_{\mathrm{SN}}^{(1)}-h}$ turns out to be $O\left[\left(h_{\mathrm{SN}}^{(1)}-h\right)^{-1 / 2}\right]$. Since in the present system, $\rho$ and $\phi$ show a limit cycle, its period $\tau$ is estimated as

$$
\tau \propto\left(h_{\mathrm{SN}}^{(1)}-h\right)^{-1 / 2}
$$

for $h$ slightly below $h_{\mathrm{SN}}^{(1)}$. Numerical result is consistent with the theoretical prediction (Fig. 12). On the other hand, slightly below the Hopf bifurcation point $h_{H}$ for $\Omega>\Omega_{c}$, by making use of the reductive perturbation method [25], the amplitude equation for the deviation of $\rho$ and $\phi$ from the limit cycle solution obeys the ordinary Ginzburg-Landautype equation

$$
\dot{w}=a w-b|w|^{2} w,
$$

$(\operatorname{Re} b>0)$, where $\operatorname{Re} a \propto h_{H}-h$ and $\operatorname{Im} a$ is identical to the characteristic frequency generated by the Hopf bifurcation, [Fig. 11(d)]. A detailed numerical analysis in the QP region far below $h_{\mathrm{SN}}^{(1)}$ and $h_{H}$, we have no clear-cut change between QP oscillations generated by the saddle-node and Hopf bifurcations. When $\gamma$ is finite but small, SBO is observed in a small $h$ region. Details are given in the following section.

\section{E. Phase diagram 5: Quasiperiodic motion for $\gamma \neq 0$}

Finally, we studied for $\gamma=0.05$. For this parameter, temporal evolutions of $\psi(t)$ are similar to those for $\gamma=0$. Figure 13 displays the numerical results for $\Omega=0.5$.

For a small anisotropy value, there exists a quasiperiodic motion as shown in Fig. 13(a). The Poincaré plot is a closed curve [Fig. 13(b)]. Because of the neutral stability of the trajectory in the tangential direction, the largest Lyapunov exponent vanishes. Figure 14 shows the bifurcation diagram and the largest Lyapunov exponent for $\gamma=0.05$ and $\Omega=0.5$. From Fig. 14, one observes that the largest Lyapunov exponent vanishes for the QP phase. Observing the bifurcation diagram and Lyapunov exponent in Fig. 14, one finds that as $h$ is decreased, the dynamical phase changes from SRO-1 to SBO-1 via the QP phase. It is observed that there exist a small window structure of limit cycle oscillations in the QP phase region. The phase diagram obtained from the Lyapunov exponent analysis is shown in Fig. 15. We find that there exists a SRO-3 phase in the QP phase. As seen from Eqs. (7) and (8), one can interpret that these limit cycles are realized as a result of the synchronization of the limit cycle motion for $\gamma=0$ under the external periodic forcing. 

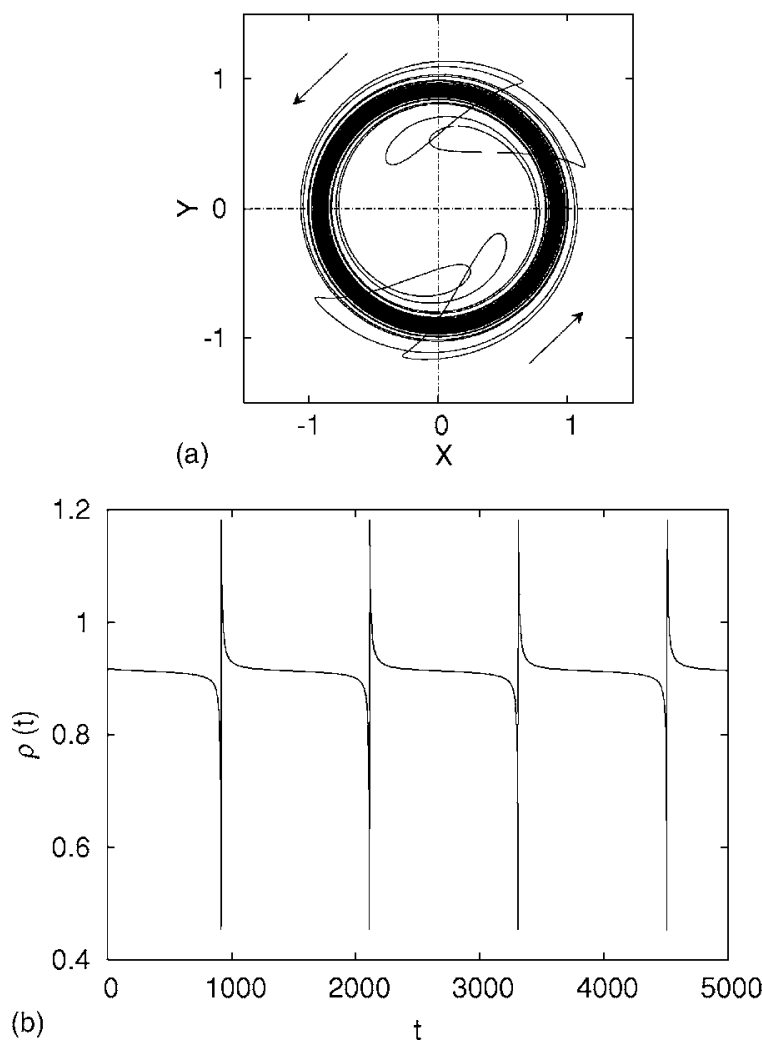
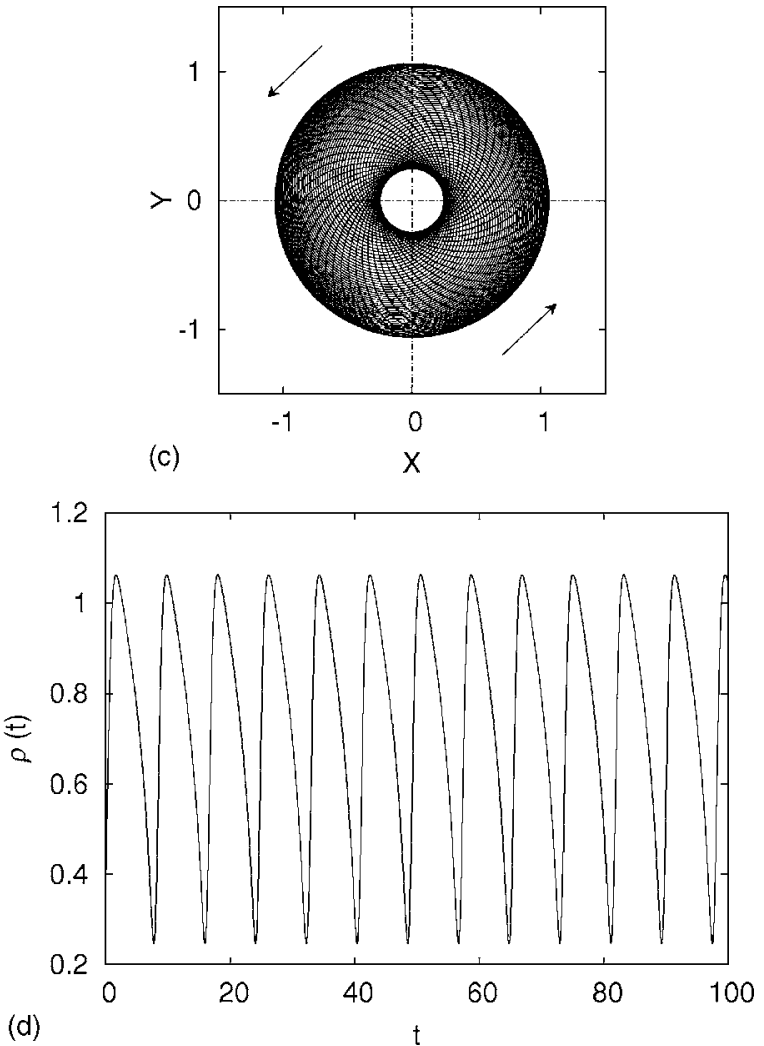

FIG. 11. QP oscillations generated via two types of bifurcations, where (a) and (c) show trajectories and (b) and (d) time evolutions of the amplitude $\rho(t)$. (a) and (b) are figures for $\gamma=0, \Omega=0.5$, and $h=0.4811$, slightly below the saddle-node bifurcation point, and (c) and (d) are for $\gamma=0, \Omega=1.0$, and $h=0.75$, slightly below the Hopf bifurcation point.

\section{CONCLUDING REMARKS}

In the present paper, we studied nonlinear dynamics of the anisotropic $X Y$ spin system under its critical temperature in a rotating external field $H(t)=h e^{i \Omega t}$. In comparison with a system in an oscillating field $H(t)=h \cos (\Omega t)$ applied in a unidirection, the rotating field excites the order parameter dynamics of both easy and noneasy axes of the system. As a result, we found new dynamical phases, quasiperiodic oscil-

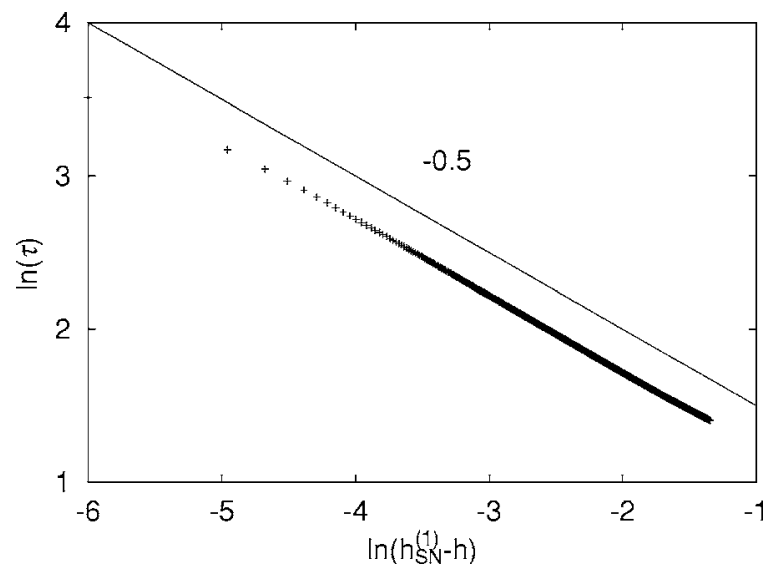

FIG. 12. Numerical results of the dependence of the period of a limit cycle $\tau$ on $\left(h_{\mathrm{SN}}^{(1)}-h\right)$ near saddle-node bifurcation point for $\gamma$ $=0$ and $\Omega=0.46$. Line in the figure corresponds to $\left(h_{\mathrm{SN}}^{(1)}-h\right)^{-1 / 2}$.

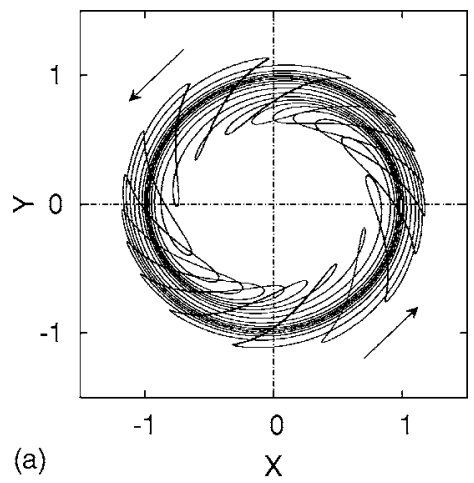

(a)

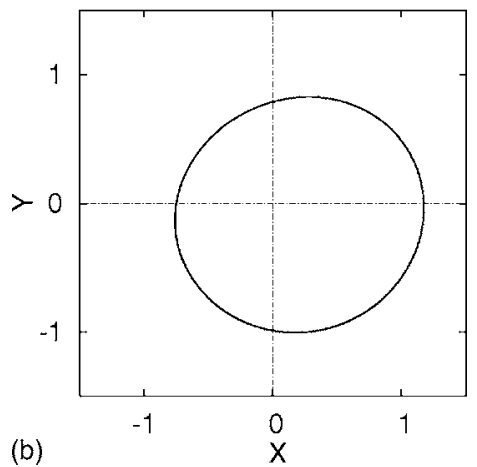

FIG. 13. (a) Quasiperiodic trajectory and (b) Poincaré plot of $\psi(n T)(n=1,2, \ldots, 10000)$ for $\gamma=0.05, \Omega=0.5$, and $h=0.40$. 

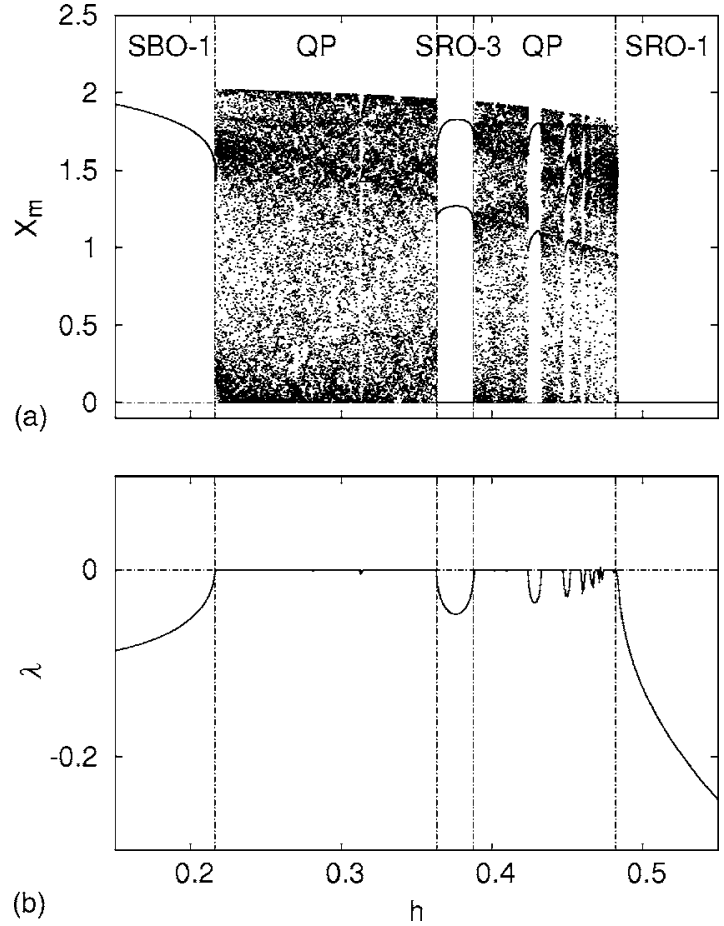

FIG. 14. (a) Bifurcation diagram and (b) the largest Lyapunov exponent $\lambda$ for $\gamma=0.05$ and $\Omega=0.5$. One observes a window structure in the QP region.

lation and chaos, which have not been reported so far in connection with dynamic phase transitions.

In Sec. III, we studied the mean-field dynamics by neglecting the spatial inhomogeneity of the order parameter. We observed both limit-cycle oscillations with multiple times of the period $T$ of the applied field and chaotic oscillations. These complicated oscillations are observed inside the SBO-1 region in the intermediate range of $\gamma$ value. We also observed quasiperiodic oscillation for small $\gamma$ value. These phenomena do not exist in a system in a linearly polarized oscillating field and are characteristic of the system in a rotating field. Furthermore, we found that there exist two

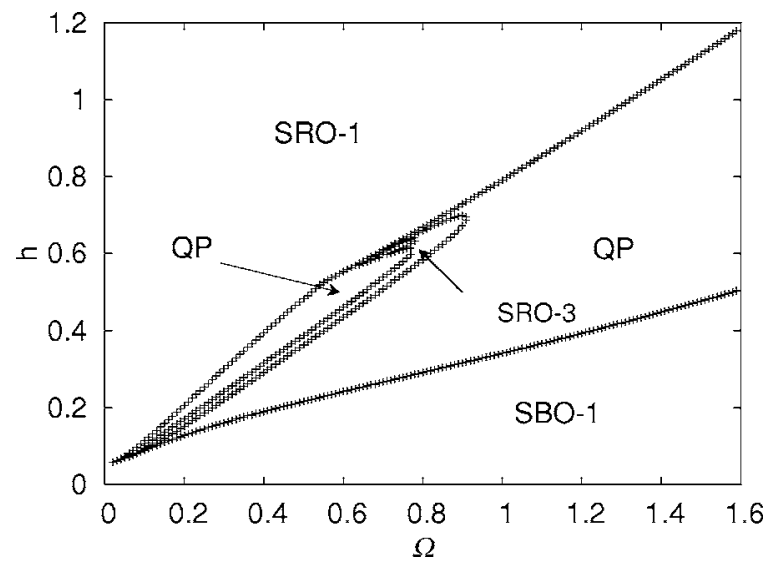

FIG. 15. Phase diagram for $\gamma=0.05$. There exist quasiperiodicity regions denoted by "QP." Furthermore, in the inner part of that region, the SRO-3 region is observed. Narrow window structure regions in $\mathrm{QP}$ are not shown in the figure. types of chaotic oscillations. Under the change of the intensity of the applied field, two symmetry-broken chaotic attractors merge and we observe one symmetric chaotic attractor. The application of external rotating periodic force on the anisotropic $X Y$ spin system was found to produce effects in contrast to that for the linearly polarized oscillating periodic force.

\section{ACKNOWLEDGMENTS}

The authors thank Per Arne Rikvold, Hiroki Tutu, Miki U. Kobayashi, and Naofumi Tsukamoto for valuable discussions. This study was partially supported by the 21 st Century COE Program "Center of Excellence for Research and Education on Complex Functional Mechanical Systems" at Kyoto University.

\section{APPENDIX A: DERIVATION OF THE SRO-1-QP TRANSITION LINE FOR THE ISOTROPIC CASE $(\gamma=0)$}

In the isotropic case $(\gamma=0)$, the fixed point solution of Eqs. (19) and (20) can be analytically obtained. This solution corresponds to the SRO-1 solution. Examining the linear stability analysis of this SRO-1 solution, one can analytically determine the transition point between SRO-1 and QP.

The fixed point solutions $\rho_{0}$ and $\phi_{0}$ satisfy Eqs. (22) and (23). Particularly, $\rho_{0}$ is determined by the crossing point of $f\left(\rho^{2}\right)$ and $h^{2}$, where $f\left(\rho^{2}\right)$ is the cubic function of $\rho^{2}$ defined in Eq. (22) (Fig. 16). Let the values of $\rho$ giving maximum and minimum values of $f\left(\rho^{2}\right)$ be $\rho_{ \pm} \equiv \sqrt{\left(2 \pm \sqrt{1-3 \Omega^{2}}\right) / 3}$. For small perturbations $\delta \rho=\rho-\rho_{0}, \delta \phi=\phi-\phi_{0}$ around the fixed point, we obtain the linearized equations of motion for $\delta \rho$ and $\delta \phi$. Setting $\delta \rho \sim e^{\lambda t}, \delta \phi \sim e^{\lambda t}$, we get $\lambda^{2}+\alpha \lambda+\beta=0$, where $\alpha=-2+4 \rho_{0}^{2}, \beta=3 \rho_{0}^{4}-4 \rho_{0}^{2}+1+\Omega^{2}$. The characteristic exponents $\lambda=1-2 \rho_{0}^{2} \pm \sqrt{\rho_{0}^{4}-\Omega^{2}}$ are complex for $\rho_{0}^{2}<\Omega$ and real for $\rho_{0}^{2} \geq \Omega$. In order that $\operatorname{Re} \lambda$ is negative, both $\alpha>0$ and $\beta>0$ must be satisfied. The condition $\alpha>0$ holds for $\rho_{0}^{2}$ $>1 / 2$. The Hopf bifurcation occurs when $\alpha$ changes its sign from positive to negative, keeping $\beta$ positive. The insertion of $\rho_{0}^{2}=1 / 2$ into Eq. (22) yields the Hopf bifurcation point $h_{H}$ given in Eq. (26).

On the other hand, the condition $\beta>0$ is satisfied if $\rho_{0}$ $>\rho_{+}$for $\rho_{-}^{2}<1 / 2$ and if $\rho_{0}<\rho_{-}$or $\rho_{0}>\rho_{+}$for $\rho_{-}^{2}>1 / 2$. As shown in Figs. 16(a)-16(c), there occurs a saddle-node bifurcation at $\rho_{0}=\rho_{+}$, namely stable and unstable fixed points collide at $\rho_{0}=\rho_{+}$. The insertion of $\rho_{0}=\rho_{+}$into Eq. (22) yields the saddle-node bifurcation point $h_{\mathrm{SN}}^{(1)}$ given in Eq. (25). In the case that the sign of $\beta$ changes at $\rho_{0}=\rho_{-}$, the saddle-node bifurcation also occurs at this point [Figs. 16(b) and 16(c)]. For $\rho_{0}=\rho_{-}$, the bifurcation point of $h$ is given by $h_{\mathrm{SN}}^{(2)}[\mathrm{Eq}$. (27)].

Henceforth, we will discuss two types of instability of the fixed point depending on $\Omega$. For $\Omega \leq 1 / 2$, the function $f$ has the form as shown in Fig. 16(a). In this case, we obtain $\Omega$ $<\rho_{-}^{2}<1 / 2<\rho_{+}^{2}$, and the fixed point is stable only when $\rho_{0}$ $>\rho_{+}$is satisfied. As $h$ is decreased, the stable fixed point disappears at $h=h_{\mathrm{SN}}^{(1)}$, where the saddle-node bifurcation occurs. A limit cycle oscillation of $\rho$ and $\phi$ is observed for $h$ $<h_{\mathrm{SN}}^{(1)}$. Since its frequency and $\Omega$ are generally incommen- 


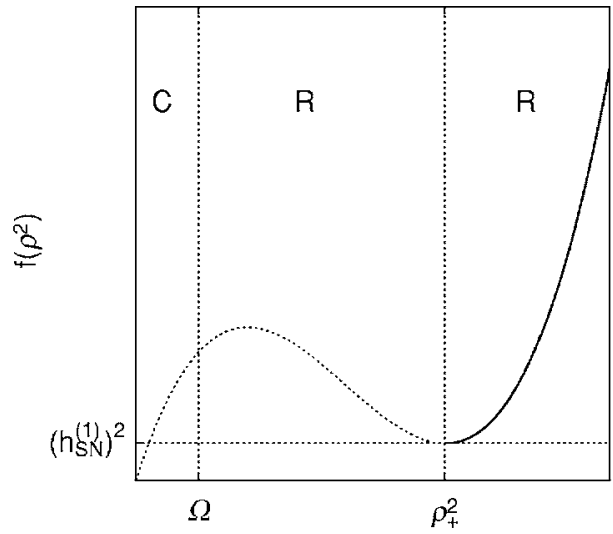

(a)

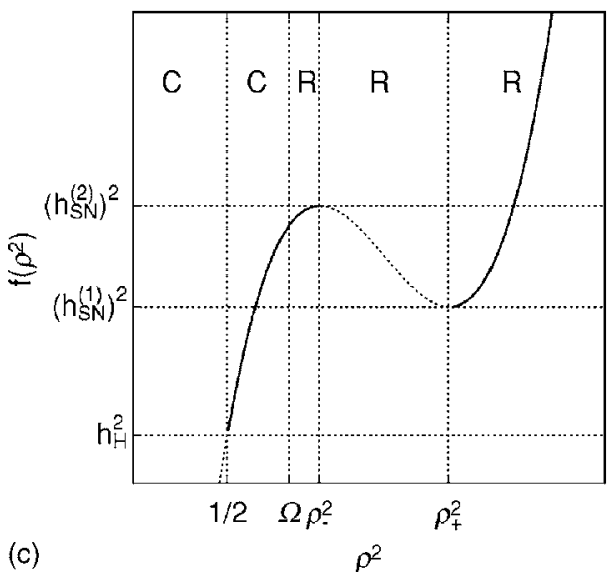

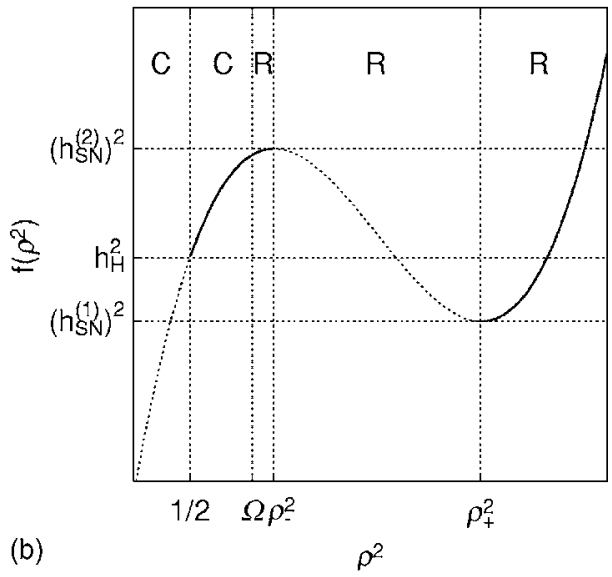

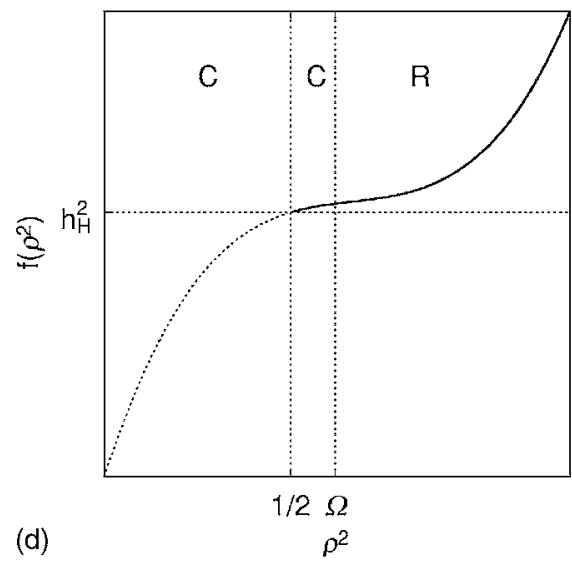

FIG. 16. Schematic figures of the function $f\left(\rho^{2}\right)$ relevant to the determination of the SRO-1 solution for (a) $\Omega \leq 1 / 2$, (b) $1 / 2<\Omega$ $<\Omega_{c}$, (c) $\Omega_{c}<\Omega<1 / \sqrt{3}$, and (d) $\Omega>1 / \sqrt{3}$. The solid and dotted lines, respectively, correspond to stable and unstable fixed points. Symbols " $R$ " (" $C$ ") implies that the characteristic exponents $\lambda$ of the linearized perturbation equations for $\delta \rho$ and $\delta \phi$ are real (complex). surate, the variable $\psi(t)$ shows a quasiperiodic oscillation.

For $1 / 2<\Omega<\Omega_{c}$, the function $f$ has the form as shown in Fig. 16(b). In this case, we get $1 / 2<\Omega<\rho_{-}^{2}<\rho_{+}^{2}$. The system shows the saddle-node bifurcation at $\rho_{0}=\rho_{+}$. In addition, for $1 / \sqrt{2}<\rho_{0}<\rho_{-}$satisfying the conditions $\rho_{0}^{2}>1 / 2$ and either $\rho_{0}<\rho_{-}$or $\rho_{0}>\rho_{+}$, i.e., in the region $h_{H}<h<h_{\mathrm{SN}}^{(2)}$, the system shows the bistability. However, even if $h$ is increased from 0 , no hysteresis is observed since the root with $\rho_{0}$ $>\rho_{+}$first comes into exist at $h=h_{\mathrm{SN}}^{(1)}$. For $\Omega_{c}<\Omega<1 / \sqrt{3}$, the function $f$ has the form as shown in Fig. 16(c). In the region $h_{\mathrm{SN}}^{(1)}<h<h_{\mathrm{SN}}^{(2)}$, the system shows the bistability. As $h$ is increased from 0 , a fixed point appears at $h=h_{H}\left(<h_{\mathrm{SN}}^{(1)}\right)$ and the hysteresis is observed. Furthermore, for $\Omega>1 / \sqrt{3}$, the function $f\left(\rho^{2}\right)$ is a monotonous function of $\rho^{2}$, and the Hopf bifurcation occurs at $h=h_{H}$ and the SRO- 1 solution becomes unstable [Fig. 16(d)].

In this way, the transition line between SRO- 1 and QP can be analytically determined for $\gamma=0$. The resulting phase diagram in the $\Omega$ - $h$ plane is shown in Fig. 10. We compared the transition line with that obtained by the numerical simulation between SRO-1 and QP and found a good agreement.

\section{APPENDIX B: PHASE DYNAMICS NEAR THE SADDLE-NODE BIFURCATION POINT FOR THE ISOTROPIC CASE $(\gamma=0)$}

Since the time evolutions of $\rho$ and $\phi$ are slow near the saddle-node bifurcation point $h=h_{\mathrm{SN}}^{(1)}$, one can neglect the $\dot{\rho}^{2}$ and $\dot{\phi}^{2}$ terms in Eq. (21) and obtains

$$
-2\left(\rho-\rho^{3}\right) \dot{\rho}+2 \rho^{2} \Omega \dot{\phi}+f\left(\rho^{2}\right)=h^{2},
$$

where $f(\xi) \equiv \xi\left[\Omega^{2}+(1-\xi)^{2}\right]$. In the following, we also neglect the $\ddot{\phi}$ term.

By defining $\rho_{*} \equiv-h \sin \phi / \Omega$, from Eq. (20) we obtain

$$
\rho(\phi, \dot{\phi})=-\frac{h \sin \phi}{\dot{\phi}+\Omega} \approx \rho_{*}\left(1-\frac{\dot{\phi}}{\Omega}\right) .
$$

Its time derivative is given by

$$
\dot{\rho} \approx-\frac{h \cos \phi}{\Omega} \dot{\phi} .
$$

We thus obtain

$$
f\left(\rho^{2}\right) \approx f\left(\rho_{*}^{2}\right)-\frac{2 \rho_{*}^{2}}{\Omega}\left[\Omega^{2}+\left(1-\rho_{*}^{2}\right)\left(1-3 \rho_{*}^{2}\right)\right] \dot{\phi} .
$$

The insertion of these into Eq. (B1) yields

$$
\dot{\phi}=\frac{1}{g(\phi, h)}\left[h^{2}-f\left(\frac{h^{2}}{\Omega^{2}} \sin ^{2} \phi\right)\right],
$$

where 


$$
g(\phi, h)=\frac{1-\rho_{*}^{2}}{\Omega}\left(-\frac{h^{2} \sin 2 \phi}{\Omega}+2 \rho_{*}^{2}\left(-1+3 \rho_{*}^{2}\right)\right) .
$$

Equation (B5) determines the slow evolution of the phase $\phi$ around $\rho_{\mathrm{SN}}$ and $\phi_{\mathrm{SN}}$, the fixed point amplitude and phase at $h=h_{\mathrm{SN}}^{(1)}$.

If $h$ is slightly above $h_{\mathrm{SN}}^{(1)}$, the equation

$$
h^{2}=f\left(\frac{h^{2}}{\Omega^{2}} \sin ^{2} \phi\right)
$$

has a linearly stable steady solution $\phi_{0}$. Expanding $f$ around $\phi=\phi_{0}$ and retaining the first order terms with respect to the deviation $\delta \phi \equiv \phi-\phi_{0}$, we obtain

$$
\dot{\delta \phi}=\frac{1}{g\left(\phi_{0}, h\right)}\left[-f^{\prime}\left(\frac{h^{2}}{\Omega^{2}} \sin ^{2} \phi_{0}\right) \frac{h^{2} \sin \left(2 \phi_{0}\right)}{\Omega^{2}}\right] \delta \phi .
$$

Here we have replaced $g(\phi, h)$ by $g\left(\phi_{0}, h\right)$. This equation shows that for $h>h_{\mathrm{SN}}^{(1)}$ the fixed point is linearly stable since $f^{\prime}>0$ and $\sin \left(2 \phi_{0}\right) / g\left(\phi_{0}, h\right)=\Omega^{2} /\left[2 h^{2}\left(2 \rho_{0}^{2}-1\right)\right]>0$ since we are concerning with the saddle-node bifurcation, i.e., $\rho_{0}^{2}$ $>1 / 2$.

On the other hand, for $h<h_{\mathrm{SN}}^{(1)}$, the stable fixed point disappears and the system exhibits a limit cycle solution, which is the onset of the quasiperiodic oscillation of $\psi(t)$. For $h$ slightly below $h_{\mathrm{SN}}^{(1)}$, the time evolution of $\phi$ near $\phi=\phi_{\mathrm{SN}}$, the fixed point value at $h=h_{\mathrm{SN}}^{(1)}$, is slow. In this case, by considering Eq. (B5) near $h=h_{\mathrm{SN}}^{(\mathrm{I})}$ and $\phi=\phi_{\mathrm{SN}}$, the slowness of the time evolution of $\phi$ is estimated as

$$
\dot{\phi}=-a\left(h_{\mathrm{SN}}^{(1)}-h\right)-b\left(\phi-\phi_{\mathrm{SN}}\right)^{2},
$$

where in terms of $g_{\mathrm{SN}} \equiv g\left(\phi_{h_{\mathrm{SN}}^{(1)}}, h_{\mathrm{SN}}^{(1)}\right)$, we have defined

$$
a=\frac{2 h_{\mathrm{SN}}^{(1)}}{g_{\mathrm{SN}}}(>0),
$$

$$
b=\frac{\sqrt{1-3 \Omega^{2}}}{\Omega^{4} g_{\mathrm{SN}}}\left(h_{\mathrm{SN}}^{(1)}\right)^{4} \sin ^{2}\left(2 \phi_{\mathrm{SN}}\right)(>0) .
$$

This equation is integrated to yield

$$
\phi(t)=\sqrt{\frac{a}{b}\left(h_{\mathrm{SN}}^{(1)}-h\right)} \tan \left[\alpha-\sqrt{a b\left(h_{\mathrm{SN}}^{(1)}-h\right)} t\right]+\phi_{\mathrm{SN}},
$$

where

$$
\alpha=\arctan \left(\frac{\phi(0)-\phi_{\mathrm{SN}}}{\sqrt{(a / b)\left(h_{\mathrm{SN}}^{(1)}-h\right)}}\right) .
$$

One finds that the characteristic time to escape from the region $\left|\phi-\phi_{\mathrm{SN}}\right|=O\left(\sqrt{h_{\mathrm{SN}}^{(1)}-h}\right)$ is $O\left[\left(h_{\mathrm{SN}}^{(1)}-h\right)^{-1 / 2}\right]$.
[1] T. Tomé and M. J. de Oliveira, Phys. Rev. A 41, 4251 (1990).

[2] J. F. F. Mendes and E. J. S. Lage, J. Stat. Phys. 64, 653 (1991).

[3] M. Acharyya, Phys. Rev. E 56, 2407 (1997).

[4] H. Fujisaka, H. Tutu, and P. A. Rikvold, Phys. Rev. E 63, 036109 (2001); 63, 059903(E) (2001).

[5] T. Yasui, H. Tutu, M. Yamamoto, and H. Fujisaka, Phys. Rev. E 66, 036123 (2002); 67, 019901(E) (2003).

[6] H. Tutu and N. Fujiwara, J. Phys. Soc. Jpn. 73, 2680 (2004).

[7] M. Keskin, O. Canko, and Ü. Temizer, Phys. Rev. E 72, 036125 (2005); O. Canko, B. Deviren, and M. Keskin, J. Phys.: Condens. Matter 18, 6635 (2006).

[8] W. S. Lo and R. A. Pelcovits, Phys. Rev. A 42, 7471 (1990).

[9] S. W. Sides, P. A. Rikvold, and M. A. Novotny, Phys. Rev. Lett. 81, 834 (1998); Phys. Rev. E 59, 2710 (1999).

[10] G. Korniss, C. J. White, P. A. Rikvold, and M. A. Novotny, Phys. Rev. E 63, 016120 (2001).

[11] G. Korniss, P. A. Rikvold, and M. A. Novotny, Phys. Rev. E 66, 056127 (2002).

[12] M. Acharyya, Phys. Rev. E 56, 1234 (1997).

[13] H. Jang and M. J. Grimson, Phys. Rev. E 63, 066119 (2001); H. Jang, M. J. Grimson, and C. K. Hall, Phys. Rev. B 67, 094411 (2003); Phys. Rev. E 68, 046115 (2003).
[14] Q. Jiang, H.-N. Yang, and G.-C. Wang, Phys. Rev. B 52, 14911 (1995).

[15] J.-S. Suen and J. L. Erskine, Phys. Rev. Lett. 78, 3567 (1997).

[16] B. K. Chakrabarti and M. Acharyya, Rev. Mod. Phys. 71, 847 (1999).

[17] M. Acharyya, Int. J. Mod. Phys. C 16, 1631 (2005).

[18] E. Machado, G. M. Buendía, P. A. Rikvold, and R. M. Ziff, Phys. Rev. E 71, 016120 (2005).

[19] G. S. Jeon, H. J. Kim, M. Y. Choi, B. J. Kim, and P. Minnhagen, Phys. Rev. B 65, 184510 (2002); G. S. Jeon, J. S. Lim, H. J. Kim, and M. Y. Choi, ibid. 66, 024511 (2002).

[20] H. Tutu, Prog. Theor. Phys. 114, 953 (2005); H. Tutu and T. Mitani, Prog. Theor. Phys. Suppl. 161, 376 (2006).

[21] M. Acharyya, Int. J. Mod. Phys. C 12, 709 (2001); 14, 49 (2003).

[22] N. Fujiwara, H. Tutu, and H. Fujisaka, Phys. Rev. E 70, 066132 (2004); Prog. Theor. Phys. Suppl. 161, 181 (2006).

[23] M. Acharyya, Phys. Rev. E 69, 027105 (2004).

[24] C. Grebogi, E. Ott, F. Romeiras, and J. A. Yorke, Phys. Rev. A 36, 5365 (1987).

[25] Y. Kuramoto and T. Tsuzuki, Prog. Theor. Phys. 54, 687 (1975). 